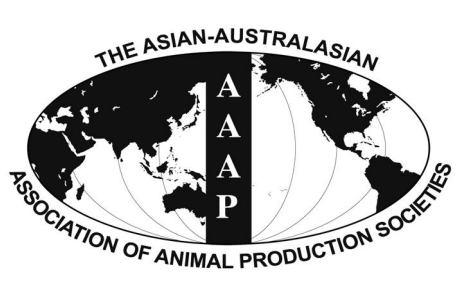

\title{
Differential Expression of miR-34c and Its Predicted Target Genes in Testicular Tissue at Different Development Stages of Swine
}

\author{
Xiaojun Zhang, Wei Zhao ${ }^{a}$, Chuanmin Li, Haibin Yu, YanYan Qiao, Aonan Li, \\ Chunyan Lu, Zhihui Zhao, and Boxing Sun* \\ College of Animal Science, Jilin University, Changchun 130062, China
}

\begin{abstract}
To verified the target genes of miR-34c, bioinformatics software was used to predict the targets of miR-34c. Three possible target genes of miR-34c related to spermatogenesis and male reproductive development: zinc finger protein 148 (ZNF148), kruppel-like factor 4 (KLF4), and platelet-derived growth factor receptor alpha (PDGFRA) were predicted. Then, the expression of miR$34 \mathrm{c}$ and its target genes were detected in swine testicular tissue at different developmental stages by quantitative polymerase chain reaction. The results suggested that the expression of PDGFRA has the highest negative correlation with miR-34c. Then immunohistochemical staining was done to observe the morphology of swine testicular tissue at 2-days and 3, 4, 5-months of age, which indicated that PDGFRA was mainly expressed in the support cells near the basement membrane during the early development stages of testicular tissue, but that the expression of PDGFRA was gradually reduced in later stages. Therefore, western blot analyzed that the highest expression of PDGFRA was generated in 2-days old testicular tissues and the expression levels reduced at 3 and 4-months old, which correlated with the results of immunohistochemical staining. In conclusion, PDGFRA is a target gene of miR-34c. (Key Words: miR-34c, PDGFRA, Testicular Tissue, Differential Expression)
\end{abstract}

\section{INTRODUCTION}

MicroRNAs (miRNAs) are endogenous, non-coding RNAs of about 20 to 25 nucleotides long that have a regulatory function in eukaryotes (Lee et al., 2004). Mature miRNAs assemble into RNA-induced silencing complexes, which target mRNA through complementary base pairing to regulate target genes by target mRNA degradation or repression of the target mRNA translation (Reinhart et al., 2000). And miRNAs play regulatory roles in many physiological processes, such as development, virus defense, hematopoiesis, organogenesis, cell proliferation and apoptosis, fat metabolism, viral replication and tumor formation (Chang et al., 2014; Mello et al., 2014; Ning et al., 2014). Numerous studies have shown that miRNAs are

\footnotetext{
* Corresponding Author: Boxing Sun. Tel: +86-431-89586105, Fax: +86-431-89586105, E-mail: sbx@jlu.edu.cn

${ }^{a}$ These two authors contributed equally to this work, and share the first authorship.

Submitted Jan. 16, 2015; Revised Apr. 13, 2015; Accepted Apr. 29, 2015
}

essential for normal spermatogenesis and male fertility.

MiR-34c, is an important miRNAs as it regulates sperm production and male fertility (Geremia et al., 1977). In mice, the expression of miR-34c is significantly different in mature and immature testicular tissues, which suggest that miR-34c could play an important regulatory role in the developmental process of male germ cells. High-throughput sequencing suggests that the expression of miR-34c also is significantly different between mature and immature swine testis (Lian et al., 2012). But few reports have investigated the target genes of miR-34c. Therefore, in the present study, the expression of miR-34c and three of its predicted target genes in Junmu No.1 swine testicular tissues at different developmental stages were investigated using quantitative polymerase chain reaction (PCR), western blot and immunohistochemical methods.

\section{MATERIALS AND METHODS}

\section{Samples}

A total of 24 samples, which included 2-days, 3-month 
to 7-month, 9-month, and 12-month old swine testicular tissue were collected from the Jilin University swine farm. Animal experiments were done under the guidance of Jilin University Animal Care and Use Committee.

\section{Bioinformatics prediction}

Three target genes of miR-34c (zinc finger protein 148: ZNF148, kruppel-like factor 4: KLF4, and platelet-derived growth factor receptor alpha: $P D G F R A$ ) were predicted by four kinds of bioinformatics software (microRNA.org, miRDB, miRGen, TargetScan). Amplification of miR-34c and target genes was done as described in the manual (Takara, Dalian, China). The cDNA sequences were retrieved from the database (http://www. Ensemble.org) and (http://www.ncbi.nlm.nih.gov/).

\section{RNA isolation, cDNA synthesis, and real-time PCR}

Swine-specific primers were designed according to mature miR-34c sequence. Primers of miR-34c and its target genes were designed for quantitative reversetranscription polymerase chain reaction according to the sequence of NCBI and miRBase14.0. U6 was used as the control (Ren et al., 2009). Primers of ZNF148 gene and reference housekeeping gene glyceraldehyde-3-phosphate dehydrogenase $(G A P D H)$ were designed as previously reported (Zhou et al., 2012). The primers for KLF4 were designed using Primer 5.0 software. All primer sequences are listed in Table 1 and were synthesized (Sangon, Shanghai, China).

Testis tissue samples from healthy swine specimens were frozen in liquid nitrogen. Trizol reagent was used to extract total RNA. The cDNA was synthesized by reverse transcription with an RT-PCR Kit (Takara) according to the instruction. The qPCR reaction mixture included cDNA (2 $\mu \mathrm{L})$, PCR-Master Mix $(10 \mu \mathrm{L})$, PCR-F-Primer $(0.5 \mu \mathrm{L})$, PCR-R-Primer $(0.5 \mu \mathrm{L})$, and RNase-free $\mathrm{H}_{2} \mathrm{O}(7 \mu \mathrm{L})$ in a total volume of $20 \mu \mathrm{L}$. The qPCR was performed in a reaction under the following procedure: $95^{\circ} \mathrm{C}$ for $30 \mathrm{~s} ; 40$ cycles of $95^{\circ} \mathrm{C}$ for $5 \mathrm{~s}$ and $60^{\circ} \mathrm{C}$ for $30 \mathrm{~s}$. The expression of miR-34c and genes were detected using the SYBR Green I (Takara) and were analyzed by Eppendorf AG-5341 fluorescence quantitative instrument. The data were analyzed using the SPSS ver. 13.0 (SPSS Inc., Chicago, IL, USA) and $2^{-\triangle \Delta C T}$ method was used according to the following formula:

$$
\begin{aligned}
\Delta \Delta \mathrm{Ct}= & {[\mathrm{Ct}(\text { positive })-\mathrm{Ct}(\text { reference })] } \\
& -[\mathrm{Ct}(\text { control })-\mathrm{Ct}(\text { reference })]
\end{aligned}
$$

Here, $2^{-\Delta \Delta \mathrm{Ct}}$ refers to the relative expression ratio.

\section{Validation of immunohistochemical staining}

To further investigate the expression of PDGFRA at different developmental stages of swine testis, immunohistochemical staining was done in testicular tissues from 2-day, 3-month, 4-month and 5-month old animals. For dewaxing and hydration, tissue sections were sequentially placed in xylene (10 minutes $\times 2), 100 \%$ ethanol (5 minutes $\times 2$ ), $90 \%$ ethanol, $80 \%$ ethanol, $70 \%$ and $50 \%$ ethanol ( 5 minutes at each concentration), distilled water (5 minutes), and 0.01 phosphate buffered saline (PBS, PH 7.4; 5 minutes $\times 3$ ). After high-pressure antigen retrieval in a pressure cooker, the tissue sections were stained with a chromogenic DAB based PDGFRA IHC kit according to manufacturer's protocol (Beijing Biosynthesis Co., Beijing, China).

\section{Western blot detection}

Total protein was extracted from testicular tissue at different developmental stages using RIPA buffer (Boster, Wuhan, China) following the manufacturer's instructions. Protein concentration was determined using the BCA

Table 1. Sequences of primers for real-time PCR

\begin{tabular}{lll}
\hline Gene & \multicolumn{1}{c}{ Primer } & \multicolumn{1}{c}{ Primer sequence $\left(5^{\prime}-3^{\prime}\right)$} \\
\hline ssc-miR-34c-5p & RT-Primer & CTCAACTGGTGTCGTGGAGTCGGCAATTCAGTTGAGGCAATCAG \\
& F-Primer & CCCGCCAGGCAGTGTAGTTA \\
& R-Primer & CTCAACTGGTGTCGTGGAGTC \\
U6 & F-Primer & CTCGCTTCGGCAGCACA \\
RNF148 & R/RT-Primer & AACGCTTCACGAATTTGCGT \\
& F-Primer & GCCTTTAGAACGAACTATCAC \\
KLF4 & R-Primer & AGGTACTTCTGTATGAAACGC \\
& F-Primer & TCGGACCACCTTGCCTTACA \\
PDGFRA & R-Primer & TTGGGAACTTGACCATGATTGTAG \\
& F-Primer & TCCAGCAGTTCTACCTTCATCA \\
GAPDH & R-Primer & AGGAGTCTATGCCAATGTCGTC \\
& F-Primer & GTTTGTGATGGGCGTGAAC \\
\hline PCR, polymimer & ATGGACCTGGGTCATGAGT
\end{tabular}

PCR, polymerase chain reaction; zinc finger protein 148(ZNF148), kruppel-like factor 4(KLF4), platelet-derived growth factor receptor alpha (PDGFRA) and glyceraldehyde-3-phosphate dehydrogenase (GAPDH). 
Protein Assay Kit (Boster). Total protein ( $35 \mu \mathrm{g}$ per sample) was resolved by sodium dodecyl sulfate polyacrylamide gel electrophoresis (SDS-PAGE) and transferred onto PVDF membrane (Bio-Red Laboratories Inc, Hercules, CA, USA). Immunoblotting was conducted using the following primary antibodies with the suggested dilutions from the manufacturer: anti-PDGFRA (Abcam, London, UK); anti- $\beta$ actin (Abcam). The antibodies were diluted with 5\% BSA (Albumin from bovine serum) and the suggested dilutions were 1:200 and 1:1,000. The immunoblots were developed using an ECL Advanced Western Blotting Detection Kit (Invitrogen, Grand Island, NY, USA).

\section{RESULTS}

The differential expression of miR-34c and its target genes in swine testes at different developmental stages

Expression of miR-34c at different developmental stages in the swine testicular tissue is shown in Figure 1. The expression of miR-34c was very low at 2-days and 3months of age, but the expression level increased significantly at 4-months and reached a plateau between 5 to 7-months of age. The expression level dropped at 9months and then came back at 12-months. The relative expression levels were significantly different between 2days and 3-months and the levels of 4, 5, 6, 7, 9, 12-months of age $(\mathrm{p}<0.01)$. However, there were no significant differences among the expression levels at 5, 6, 7, 9, 12months of age ( $p>0.05)$.

The relative expression level of ZNF148 was gradually upregulated from 2-days to 6-months old. The expressions were down regulated from 7-months to 9-months, but upregulated again at 12-months. The relative expression levels before 5 -months were significantly different from the expression levels of 6,7 , and 12 -months $(p<0.05)$. No significant differences existed among the expression levels at $4,5,6,7,9$, and 12-months ( $\mathrm{p}>0.05$ ).

For KLF4, the expressions were relatively low and stable from 2-days to 4-months, but significantly increased to the peak value at 5 -months. The expression was down regulated from 6-months to 9-months and upregulated again at 12-months. The results showed that the relative expression levels at 2-days, 3-months, and 4-months were significantly different from the level at 5 -months $(\mathrm{p}<0.05)$. No significant differences exist among the expression levels at 5, 6, 7, 9 and 12 months of age ( $>00.05$ ).

For PDGFRA, the expression reached the highest level at 2-days and then down regulated to the lowest level at 4 months. The expression levels maintained relatively lower from 5-months to 12 -months.

\section{Location expression of PDGFRA gene in testis}

As shown in Figure 2A, PDGFRA is highly expressed in the surrounding cells of the spermatic basement membrane (including Sertoli cells and spermatogonial stem cells) in 2-days testicular tissues. A small amount of PDGFRA was also expressed in Leydig cells and mesenchymal cells.

Also, Figure 2B to 2D show the expression of PDGFRA in 3-months to 5-months swine testicular tissues. The locations of expressions were similar to 2-days testicular tissue, but the staining signals were weaker.

\section{The protein of $P D G F R A$ gene differentially expresses in swine testes at different developmental stages}

As shown in Figure 3, the protein of PDGFRA gene was differentially produced at different developmental stages of testis, which was detected by western blot. The highest expression level was at swine testicular tissue of 2-days old, and gradually decreased from 3-months to 4-months.
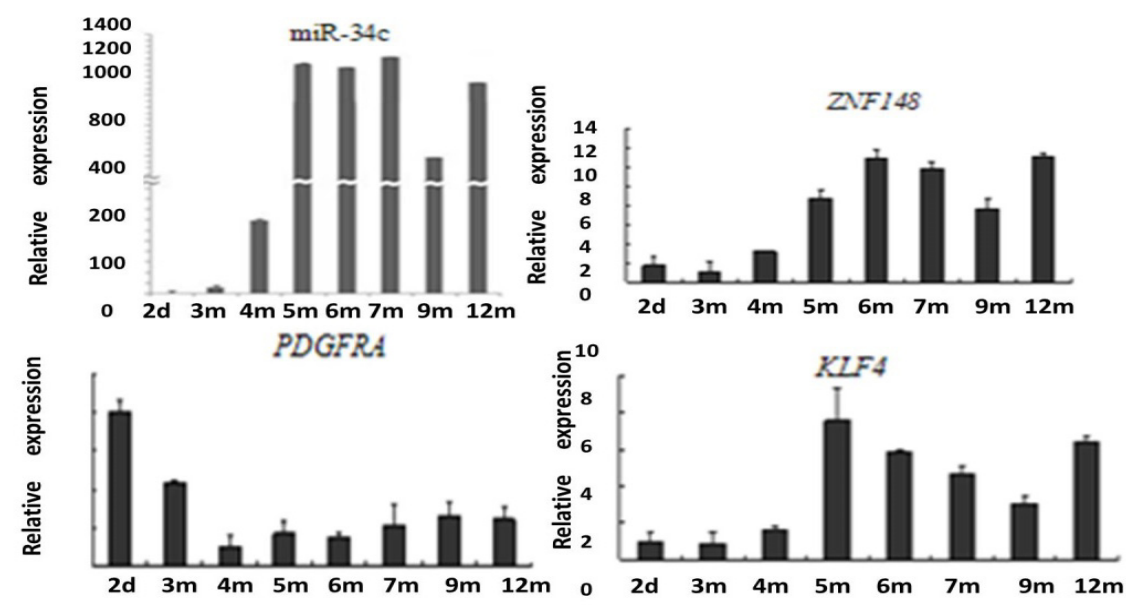

Figure 1. mRNA expressions of miR-34c and target genes In this Figure, we detected the expressions of miR-34c and target genes zinc finger protein $148(Z N F 148)$, kruppel-like factor 4 (KLF4), and platelet-derived growth factor receptor alpha $(P D G F R A)$ in swine testis at 2-days, 3- to 7-month-old, 9-month-old, and 12-month-old by qPCR. 


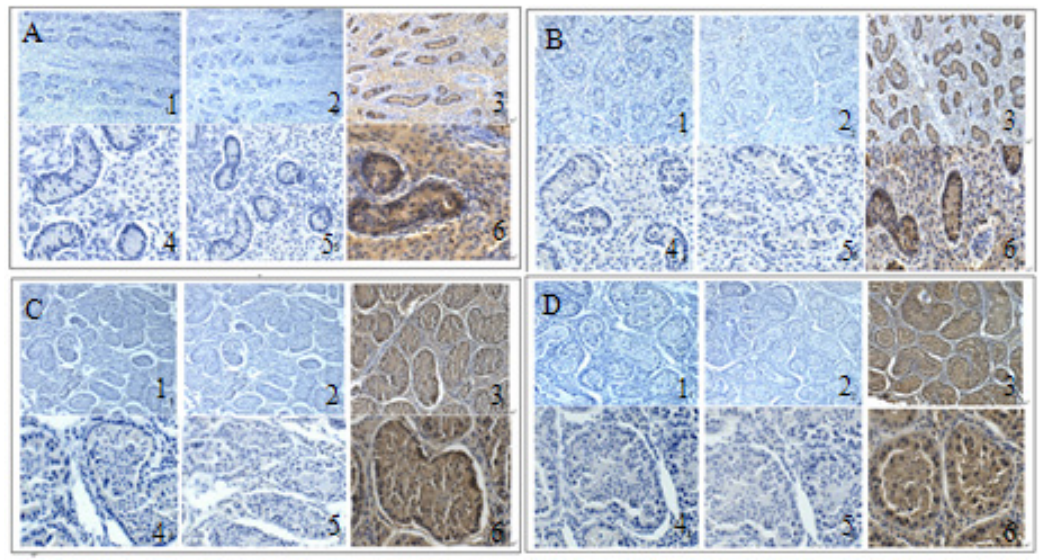

Figure 2. Tissue sections of immunohistochemical staining. In this Figure, immunohistochemical staining was done about $P D G F R A$ gene in testicular tissues at 2-days, 3-month-old, 4-month-old, and 5-month-old. (A) Two days swine testis. (B) Three months swine testis. (C) Four months swine testis. (D) Five months swine testis. And (1 to 3$)$ respectively represent testis observations $(\times 100)$, also (4 to 6$)$ respectively represents testis observations under $(\times 400)$. $(1,4)$ were treated using PBS, $(2,5)$ were treated using immune serum as negative control, $(3,6)$ used PDGFRA-specific antibody. PDGFRA, platelet-derived growth factor receptor alpha.

However, the expression level upregulated again at 5months of age.

\section{DISCUSSION}

miR-34c is involved in many important biological processes, such as the early development of somatic cell nuclear transfer bovine embryos, as a putative tumor suppressor in high-grade serous ovarian cancer, growth and invasion of colorectal cancer cells and the permeability of blood-tumor barrier. It is also required for spermatogenesis, but not for the first cleavage division in mice. Studies have shown that miR-34c has a significantly higher expression in mature mouse testis than in immature testis (Yan et al., 2007; Yoshida et al., 2007), suggesting that miR-34c plays an important role in germ cell development (Sette et al., 2000; Bouhallier et al., 2010). At the same time, studies have shown that miR-34c plays important role in these processes by regulating the target genes. So it is extremely valuable to predict and verify the targets of miR-34c.

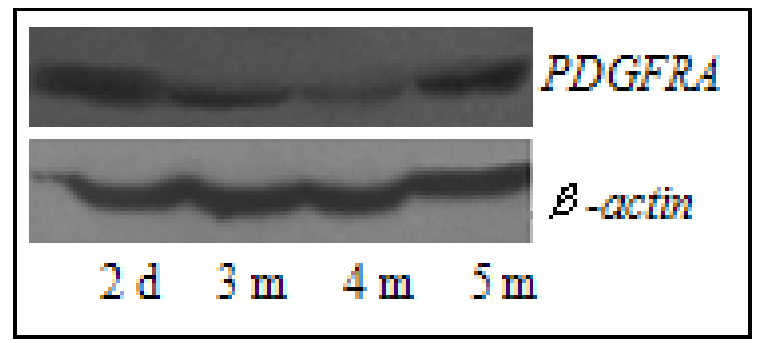

Figure 3. Protein expression of PDGFRA gene. In this Figure, the protein expression of PDGFRA gene was detected by western blot in testicular tissues at 2-days, 3-month-old, 4month-old and 5-month-old. PDGFRA, platelet-derived growth factor receptor alpha.
The bioinformatics database, is an important tool in predicting target genes, which can be assessed through matching the degree of conformation between the sequence of miRNAs and UTR sequence of genes. Based on this principle, we predicted three target genes (ZNF148, KLF4, and PDGFRA) of miR-34c regarding spermatogenesis and male fertility.

Then, we detected the expression of miR-34c and targets at different development stages of testicular tissue. Our results show that miR-34c was more highly expressed in swine testicular tissues after 4-months of age. While the predicted target gene of miR-34c, PDGFRA, was highly expressed in 2-days old testicular tissues. The expressions of KLF4 were relatively low and stable from 2-days to 4months, but significantly increased to the peak value at 5months. The relative expression level of ZNF148 was gradually upregulated from 2-days to 6-month old. SPSS ver. 13.0 (SPSS Inc., Chicago, IL, USA) analyzed that the expression of ZNF148 and KLF4 were positively correlated with the expression of miR-34c. However, miR-34c had a significant negative correlation with $P D G F R A(\mathrm{R}=-0.6$, $\mathrm{p}<0.001$ ), suggesting that $P D G F R A$ gene may be a true target gene of miR-34c. Furthermore, the detected protein expression of PDGFRA show that it is differentially expressed at different developmental stages of testis, which is consistent with the results detected by fluorescence quantitative PCR. The following results of immunohistochemistry sections showed that the expression levels of PDGFRA in 2-days and 3-month were significantly higher than in 4-months and 5-months, which is consistent with the earlier results and provided further evidence that PDGFRA was a target gene of miR-34c. In addition, the expression of miR-34c was upregulated rapidly indicating that mi-34c was important for the growth 
of seminiferous tubules. While, the expression of PDGFRA was mainly in cells surrounding the basement membrane, mainly in Sertoli cells, indicating that PDGFRA, as a target gene of mi-34c, may play an important role in the development of Sertoli cells in testis during early development stages.

\section{CONFLICT OF INTEREST}

We certify that there is no conflict of interest with any financial organization regarding the material discussed in the manuscript.

\section{ACKNOWLEDGMENTS}

This study was supported by grant from the Science and Technology Development Program in Jilin Province, Item Number (20140204067NY). And grant from Science and Technology Development Program of Changchun, Item Number (12XN28).

\section{REFERENCES}

Bouhallier, F., N. Allioli, F. Lavial, F. Chalmel, M. H. Perrard, P. Durand, J. Samarut, B. Pain, and J. P. Rouault. 2010. Role of miR-34c microRNA in the late steps of spermatogenesis. RNA 16:720-731.

Chang, R. C., W. Ying, F. W. Bazer, and B. Zhou. 2014. MicroRNAs control macrophage formation and activation: The inflammatory link between obesity and cardiovascular diseases. Cells 3:702-712.

Geremia, R., C. Boitani, M. Conti, and V. Monesi. 1977. RNA synthesis in spermatocytes and spermatids and preservation of meiotic RNA during spermiogenesis in the mouse. Cell Differ. 5:343-355.
Lee, Y., M. Kim, J. Han, K. H. Yeom, S. Lee, S. H. Baek, and V. N. Kim. 2004. MicroRNA genes are transcribed by RNA polymerase II. EMBO 23:4051-4060.

Lian, C., B. Sun, S. Niu, R. Yang, and Z. Zhao. 2012 A comparative profile of the microRNA transcriptome in immature and mature porcine testes using Solexa deep sequencing. FEBS J. 279:964-975.

Mello, T. R., A. C. Aleixo, D. G. Pinheiro, F. M. Nunes, M. M. Bitondi, K. Hartfelder, A. R. Barchuk, and Z. L. Simões. 2014. Developmental regulation of ecdysone receptor (EcR) and EcR-controlled gene expression during pharate-adult development of honeybees (Apis mellifera). Front Genet 5:445. Ning, Z., H. Zhu, F. Li, Q. Liu, G. Lig, T. Tan, B. Zhang, S, Chen, G. Li, D. Huang, S. J. Meltzer, and H. Zhang. 2014. Tumor suppression by miR-31 in esophageal carcinoma is p21dependent. Genes Cancer 5:436-440.

Reinhart, B. J., F. J. Slack, M. Basson, A. E. Pasquinelli, J. C. Bettinger, A. E. Rougvie, H. R. Horvitz, and G. Ruvkun. 2000. The 21-nucleotide let-7RNA regulates developmental timing in Caenorhabditis elegans. Nature 403:901-906.

Ren, J., H. Tang, X. Yan, X. Huang, B. Zhang, H. Ji, B. Yang, D. Milan, and L. Huang. 2009. A pig-human comparative RH map comprising 20 genes on pig chromosome 13q41 that harbours the ETEC F4ac receptor locus. J. Anim. Breed Genet. 126:30-36.

Sette, C., S. Dolci, R. Geremia, and P. RossiP. 2000. The role of stem cell factor and of alternative c-kit gene products in the establishment, maintenance and function of germ cells. Int. J. Dev. Biol. 44:599-608.

Yan, N., Y. Lu, H. Sun, D. Tao, S. Zhang, W. Liu, and Y. Ma. 2007. A microarray for microRNA profiling in mouse testis tissues. Reproduction 134:73-79..

Yoshida, S., Y. Nabeshima, and T. Nakagawa. 2007. Stem cell heterogeneity: actual and potential stem cell compartments in mouse spermatogenesis. Ann. N Y Acad. Sci. 1120:47-58.

Zhou, Y., Y. Liu, and L. Cheng. 2012. miR-21 expression is related to particle-induced osteolysis pathogenesis. J. Orthop. Res. 30:1837-1842. 